This is a postprint version of the following published document:

Albalá, R., Olmos, D., Aznar, A. J., Baselga, J. \&

González-Benito, J. (2004). Fluorescent labels to

study thermal transitions in epoxy/silica

composites. Journal of Colloid and Interface

Science, 277 (1), pp. 71-78.

DOI: $10.1016 /$ j.jcis.2004.04.018

(C) Elsevier, 2004

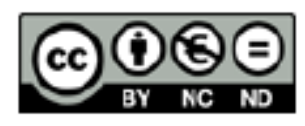

This work is licensed under a Creative Commons Attribution-NonCommercialNoDerivatives 4.0 International License. 


\title{
Fluorescent labels to study thermal transitions in epoxy/silica composites
}

\author{
R. Albalá ${ }^{\mathrm{a}}$, D. Olmos ${ }^{\mathrm{a}}$, A.J. Aznar ${ }^{\mathrm{b}}, \mathrm{J}_{\text {. Baselga }}^{\mathrm{b}}$, J. González-Benito ${ }^{\mathrm{b}, *}$ \\ a Materials Science Department, Universidad Carlos III de Madrid, Madrid, Spain \\ ${ }^{\mathrm{b}}$ Institute of Chemistry and Materials Álvaro Alonso Barba, Avda. Universidad, 30, 28911 Leganés, Madrid, Spain
}

\begin{abstract}
The analysis of the fluorescen response from the dansyl moiety as a function of temperature has been used to estimate the thermal transi-tions in silica particle/epoxy-based composite materials. Silica particles were surface-coated with 3aminopropyltriethoxysilane (APTES) and 3-aminopropylmethyldiethoxysilane (APDES). 5-Dimethylamino-1naphtalenesulfonyl chloride (DNS) and 5-dimethylaminonaphthalene-1-(2-aminoehyl)) sulfonamide (DNS-EDA) were selectively attached to the silanized silica particles and to the epoxy matrix respectively. The fluoresce ce results, interpreted in terms of the model of interpenetrating polymer networks, suggest that (i) independent of the silane coating, the interfacial region is slightly more rigid and heterogeneous than the epoxy bulk and (ii) the interface generated with APTES seems to be more flexibl than that obtained with APDES.
\end{abstract}

Keywords

Fluorescence; Thermal transitions; Composites; Silane coatings

\section{Introduction}

It is well known that the properties of composite materials are greatly affected by the kind of adhesion between their constituents [1-3]. They mainly depend on the ability of the interface to transfer stresses between the matrix and the re-inforcement [4]. In the case of polymer reinforced or fille with silica substrates (glass fibers silica particles, etc.), the addition of silane coupling agents to modify the surface of the fille is the most common method to improve the ad-hesion between the polymer matrix and the reinforcement. Additionally, the mechanical properties of these compos-ites can considerably change as a consequence of different structures that are generated from those coupling agents at the interface [4-6]. There have been several attempts to ex-plain the mechanisms that improve the mechanical proper-ties [2,4]. However, in many cases the interface is not as well known as one would like. Due to this, for these systems, it is necessary, among other things, to know the dynamic behavior or any magnitude related with it, for instance the glass transition temperature in the interfacial region. It is

* Corresponding author. Fax: +34-1-624-9430.

E-mail address: javid@ing.uc3m.es (J. González-Benito). reasonable to think that the transition, in terms of the mechanical properties, from the reinforcement to the polymer matrix should be as smooth as possible in order to optimize the stress transfer between them. For instance, in terms of rigidity it should be interesting to avoid a sharp transition between the reinforcement and the matrix. Therefore, the interface should have intermediate properties with respect to the properties of the constituents of the composite. One way to modify the interfacial properties may be by changing the molecular structure of the coupling region, for example, by changing the molecular structure of the surface coating in the reinforcement if a silane has been used as the coupling agent. However, due to the low weight fraction of the coupling region, the conventional techniques of thermal analysis are not adequate to obtain thermal transitions at the interface.

Recently Van Assche and Van Mele [7], using microthermal analysis with an AFM, were able to determine the glass transition temperatures as a function of the distance to the surface of the reinforcement in a silica/epoxy com-posite in the microscale. Although that method opens a new window on the interface world, some important as-pects should be improved. First of all, sample preparation is expensive and difficult second, since the samples must be 
cut for AFM inspection, there is no clear evidence that this process was not going to affect the sample properties; and, f nally, local or molecular-scale information cannot be obtained.

Due to their high sensitivity and site specificit, the use of $f$ uorescent probes and labels could be a good alternative. In general, luminescent methods are based on the dependence of the fuorescent emission intensity on temperature (radiative and nonradiative constant rates) when that dependence is related to the thermal relaxation processes of the polymer [8-15]. For example, Atvars et al. [12] obtained the glass transition temperature in poly(ethylacrylate) networks using anthracene as a fluore cent probe and as a label. Furthermore, with the same method they studied the secondary relaxation processes assigned to rotation of small polymer segments [13]. However, these fuorescent methods do not eliminate the effect of intensity variations arising from external factors such as lamp intensity, optical alignment, probe location, and excitation area.

Lenhart et al. [16], analyzing the band shift of dimethylaminonitrostilbene (wavelength at the maximum of the emission band) as a function of temperature, studied an apparent glass transition of the interfacial region formed by a glass microscope coverslit and an epoxy polymer. However, it would be interesting to transfer this kind of study to real systems as glass fi ers or silica-particle-reinforced composites, modifying the coupling region in a controlled way and studying the effect. Furthermore, other f uorescent probes and labels and new photophysical parameters should be found in order to clarify the mechanism responsible for the fuorescence variations when polymer relaxation is taking place.

A good alternative could be to use the same photophysical parameter proposed by our group to accurately monitor polymerizations $[17,18]$. This method is based on the f rst moment of the f uorescence band, $\langle v\rangle=\Sigma I_{F}\left(v_{F}\right) v_{F} /$ $\Sigma I_{F}\left(v_{F}\right)$, where $I_{F}\left(v_{F}\right)$ is the fuorescent intensity at a specif $\mathrm{c}$ wavenumber.

It is known that the emission from dansyl and its derivatives shows considerable variation in the Stokes shift as a function of its surroundings in terms of polarity and/or rigidity (mainly associated with free volume variations) [17-21]. Therefore, these chromophores can be a good choice in order to study, from their f uorescence variations, changes in the free volume of a polymer system as they happen in glass transition phenomena.

In this work, to estimate the thermal transitions in epoxybased composite materials, the analysis of the fuorescent response from the dansyl moiety as a function of temperature is proposed. The dansyl group will be chemically attached to the epoxy matrix in order to obtain its thermal transition and it will be compared with that obtained by a conventional technique, differential scanning calorimetry (DSC). Additionally, in order to study the thermal transitions at the interface and compare them with those obtained in the polymer matrix, the fuorescent molecule will be chemically bonded exactly at the coupling region of the composite. Different silane coupling agents will be used to modify the surface of the reinforcement and therefore to generate different structures of the coupling region. The effect of the silane coating on the thermal relaxations at the interface will be then discussed.

\section{Experimental}

\subsection{Materials}

Silica particles, SMF2, supplied by Tolsa (Madrid), were used as the fller in the composites studied in this work. These particles have approximately $95 \%(w / w) \mathrm{SiO}_{2}$ and a specifi surface of $500 \mathrm{~m}^{2} / \mathrm{g}$. The particles have a needlelike shape with a nanometric diameter and a micrometric length. However, in the system under study they form agglomerates without any specif $\mathrm{c}$ shape and sizes of tens of micrometers [22]. Furthermore, the silica agglomerates have a mesoporous structure.

Two silane coupling agents (Fluka-Chemika) were used to modify the surface of the silica particles: 3-aminopropyltriethoxysilane (APTES), I, and 3-aminopropylmethyldiethoxysilane (APDES), II:

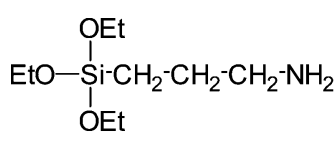

I<smiles>CCO[Si](C)(CC)OCC</smiles>

II
As f uorescent labels two dansyl derivatives were used: 5-dimethylamino-1-naphtalenesulfonyl chloride (DNS), III, to label the silica particles (Lancaster, Inc.), and 5-dimethylaminonaphthalene-1-(2-aminoehyl)) sulfonamide (DNSEDA), IV, to label the epoxy resin (synthesized and characterized in our laboratory):

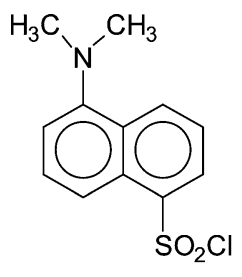

III

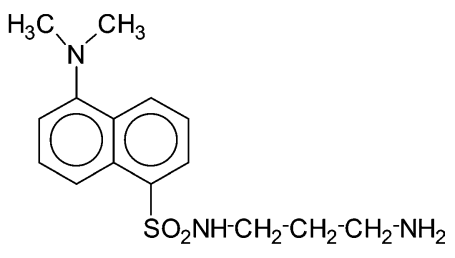

IV
The components of the polymeric system poly (Bisphenol A-co-epichlorohydrin) glycidyl end-capped (DGEBA), V, $\left\langle M_{n}\right\rangle=348 \mathrm{~g} / \mathrm{mol}(n=0.03)$, and ethylenediamine (EDA), VI, were purchased from Aldrich Co.: 


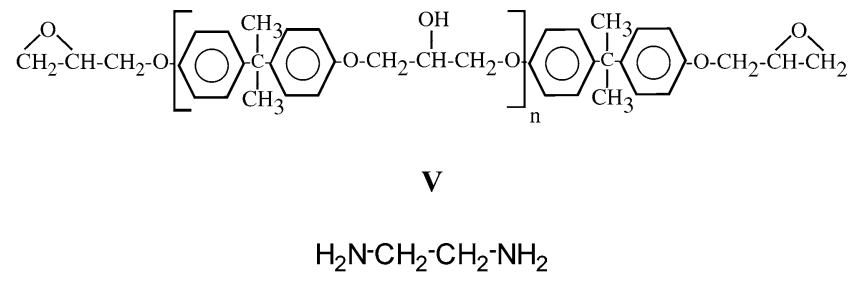

VI

\subsection{Sample preparation}

\subsubsection{Silanization}

Silica particles, g, were immersed in $50 \mathrm{ml}$ of a $2 \%(\mathrm{v} / \mathrm{v})$ silane aqueous solution for $15 \mathrm{~min}$. After that, the silica particles were fltered, washed with distilled water, and introduced into an oven at $110^{\circ} \mathrm{C}$ for $1 \mathrm{~h}$ to polymerize the silane. Finally, the silica particles were Shoxlet extracted with dry toluene distilled over sodium for $4 \mathrm{~h}$ in order to eliminate any physisorbed residue and vacuum-dried at room temperature for $14 \mathrm{~h}$. Two aqueous silane solutions were used to generate different molecular structures in the coupling region of the composite: one with APTES and the other with APDES.

\subsubsection{Dansylation}

To label APTES and APDES, silica coatings with dansyl groups, $0.4 \mathrm{~g}$ of silanized silica particles were immersed in a $1.0 \times 10^{-4} \mathrm{M}$ solution of DNS in acetonitrile $(\mathrm{AcN})$ for $15 \mathrm{~min}$. Sulfonamide formation by nucleophilic substitution of sulfonyl chloride with primary and secondary amines [23] is the mechanism on which the Hinserg's test to distinguish between primary, secondary and tertiary amines is based. The sulfonyl group of the dansyl moiety reacts easily with primary amine functionality to yield a sulfonamide fl orescent derivative [24]. One possible structure of the f nal silane product of the labeling reaction when there is reaction with the amine groups coming from the aminosilane coating may be represented by VII:

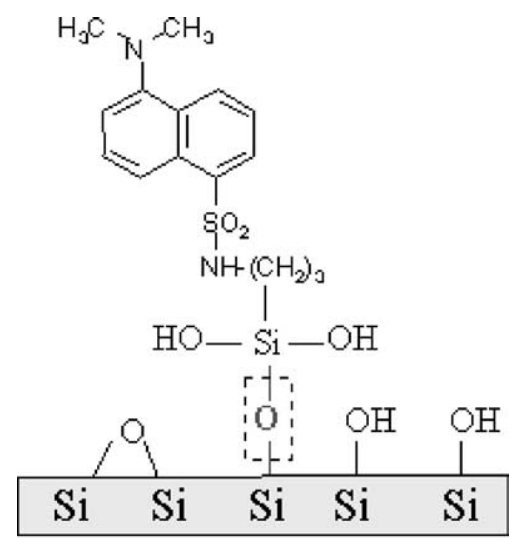

Table 1

Samples codes, type of silane coating, and dansyl group localization

\begin{tabular}{lll}
\hline Sample & Coating & Dansyl group localization \\
\hline APTES-B & APTES & Epoxy bulk \\
APTES-I & APTES & Interface \\
APDES-B & APDES & Epoxy bulk \\
APDES-I & APDES & Interface \\
\hline
\end{tabular}

After that, the dansyl-labeled silica particles were exhaustively washed with AcN. The epoxy matrix was dansyllabeled by treating the DGEBA with the reactive derivative DNS-EDA at $60^{\circ} \mathrm{C}$ for $6 \mathrm{~h}$ following the procedure already described [25].

\subsubsection{Composite preparation}

The stoichiometric mixture of DGEBA and EDA were blended with the silica particles in a proportion of $20 \%$ $(w / w)$. Two kind of samples were prepared (Table 1): one of them with the dansyl group exclusively attached to the epoxy matrix (study of thermal transitions of the epoxy bulk) and the other with the dansyl group chemically bonded to the silane coating (study of thermal transitions of the interface).

\subsection{Experimental techniques}

\subsubsection{FT-NIR}

Before the sample preparation, using FT-IR spectroscopy in the near range (FT-NIR), the epoxy-curing conditions to reach nearly $95 \%$ conversion in terms of epoxy group reaction were selected for every sample. These curing conditions were based on two steps: (i) $60^{\circ} \mathrm{C}$ for $6 \mathrm{~h}$ and (ii) postcuring at $150^{\circ} \mathrm{C}$ for $2 \mathrm{~h}$. The curing reaction was monitored by placing the composite sample between two microscope glass plates with an optical path of approximately $0.6 \mathrm{~mm}$ thickness (determined by the thickness of a Teflo spacer) and using a SPECAC temperature controller. The IR spectra in the near range (resolution $4 \mathrm{~cm}^{-1}$ and 10 scans) were recorded in a FT-IR Spectrum GX (Perkin-Elmer) using a homemade program to collect spectra as a function of time. The extent of reaction, $\alpha$, at any time, $t$, obtained from the FT-NIR spectra is calculated in terms of the epoxy group absorption according to the equation

$\alpha=1-\frac{\left(A_{E, t}\right)\left(A_{R, 0}\right)}{\left(A_{E, 0}\right)\left(A_{R, t}\right)}$,

where, $A_{E, 0}$ and $A_{R, 0}$, are the initial areas of the epoxy and reference bands, respectively, and $A_{E, t}$ and $A_{R, t}$ their values at a given time $t$. The area of the band centered at $4530 \mathrm{~cm}^{-1}$ was used to monitor the disappearance of the epoxy groups, while the band at $4623 \mathrm{~cm}^{-1}$ due to a combination band $\mathrm{C}-\mathrm{H}$ stretching vibration of the benzene ring was used as the reference $[26,27]$. 


\subsection{2. $D S C$}

The glass transition temperature for every sample was determined from the typical dynamic DSC thermogram of a Mettler Toledo DSC $822^{\mathrm{e}}$ scanning from 40 to $200^{\circ} \mathrm{C}$ with a heating rate of $20^{\circ} \mathrm{C} / \mathrm{min}$ in a nitrogen atmosphere.

\subsubsection{Fluorescence}

Fluorescence spectra were recorded as a function of temperature in an Edinburgh Instruments Co. f uorimeter using an optical fi er cable to both excite and collect in situ the $\mathrm{fl}$ orescence of the sample. The excitation and emission slits were set at 0.45 and $4.5 \mathrm{~nm}$, respectively. Every spectra were recorded between $370-710 \mathrm{~nm}$, setting the excitation wavelength at $360 \mathrm{~nm}$. The fully cured samples were placed in the SPECAC temperature controller exactly as was done in the FT-NIR equipment and the spectra were recorded as a function of temperature every $5^{\circ} \mathrm{C}$ with at least $5 \mathrm{~min}$ for thermal stabilization.

\section{Results and discussion}

\subsection{Study of thermal transitions by fluo escence}

In Fig. 1, as an example, the spectra of the dansyl group chemically bonded to the silica/epoxy interface in the sample APTES-I are represented at different temperatures; the rest of samples shown a similar behavior. When the temperature increases, two phenomena can be observed (Fig. 1): (i) the integrated intensity decreases and (ii) the emission band shifts to longer wavelength (red shift).

The frst observation can be interpreted in terms of an increase in the nonradiative processes represented by an enhancement of the nonradiative rate constant, $k_{\mathrm{nr}}$.

Generally, the solvatochromic and thermochromic shifts observed in the fuorescent emission of some fuorophores

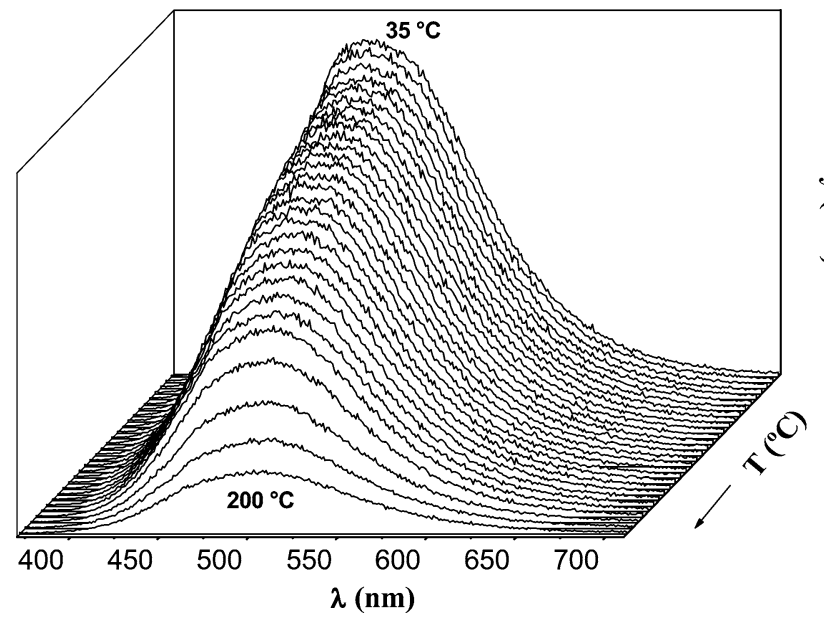

Fig. 1. Fluorescent spectra of the dansyl group chemically bonded to the silica/epoxy interface (sample APTES-I) at different temperatures. can be explained by means of the Lippert equation,

$$
\begin{aligned}
\Delta v & =v_{A}-v_{F} \\
& =\frac{2\left(\mu_{e}-\mu_{g}\right)^{2}}{h c a^{3}}\left[\frac{(\varepsilon-1)}{(2 \varepsilon+1)}-\frac{\left(n^{2}-1\right)}{\left(2 n^{2}+1\right)}\right]+\text { constant }
\end{aligned}
$$

or the equation

$\frac{\Delta v_{F}}{\Delta T}=\frac{\left(\mu_{e}-\mu_{g}\right)^{2}}{4 \pi \varepsilon_{0} h c a^{3}} \frac{\Delta\left(f(\varepsilon)-f\left(n^{2}\right)\right)}{\Delta T}$,

where $v_{A}$ and $v_{F}$ are the wavenumbers for the maxima in the absorption and emission spectra, respectively; $\mu_{e}$ and $\mu_{g}$ the dipole moments for the excited and ground states, respectively; $\varepsilon$ the dielectric constant, $n$ the refraction index, $a$ the cavity radius where the chromophore is immersed, $h$ the Planck constant, $c$ the speed of light, $\varepsilon_{0}$ the permittivity of vacuum, and $T$ the absolute temperature. Furthermore,

$f(\varepsilon)=\frac{(\varepsilon-1)}{(2 \varepsilon+1)} \quad$ and $\quad f\left(n^{2}\right)=\frac{\left(n^{2}-1\right)}{\left(2 n^{2}+1\right)}$.

Equations (2), (3) and (4) reflec the fact that the solvatochromic and thermochromic shifts mainly depend on changes in the physical properties of the solvent $(\varepsilon, n$, and viscosity) [28,29]. In a low-viscosity solvent, there usually is a blue shift when the temperature increases [29]. This aspect is apparently opposite to the results observed in Fig. 1, where a red shift is shown. However, for this case it is necessary to take into account that if the polymeric matrix is considered as the solvent of the fuorescent moiety, the solvent relaxation should be very restricted due to the high viscosity of the medium. In rigid systems, such as polymers below the glass transition temperature, the thermochromic shifts usually are batochromic [30-32].

There are several phenomena that could lead the spectral changes observed in Fig. 1, induced by variations in temperature: (i) a decrease in the solvent relaxation time; (ii) changes in the dielectric constant of the solvent; (iii) changes in the refraction index of the solvent; and (iv) an increase in the population of the upper vibrational levels of the DNS's electronic ground state (thermal activation).

A decrease in the solvent relaxation time should lead to more effective dipolar coupling between the solvent dipoles and the f uorophore excited state. This effect would yield greater excited-state stabilization and therefore a red shift [33]. However, it does not seem reasonable, in a rigid medium, to justify the batochromic shifts in terms of the solvent relaxation mechanism, since the dipoles reorientation time must be much longer than the fuorescence lifetime of the DNS's excited state. Thus, there is low probability for the excited state to be stabilized by dipole coupling [33,34].

The absence of the typical thermochromic effect (blue shift) can be justifie in terms of Eq. (2). Most of the physical properties of polymers (heat capacity, thermal expansion coeff cient, storage modulus, refraction index) show a discontinuity at the glass transition. In general, for a polymer 
system, at temperatures lower than the glass transition temperature, $T_{g}$, the dielectric constant is nearly constant with temperature $[35,36]$. On the other hand, since the refraction index, $n$, is directly related with changes in the density of the medium $[37,38]$, is reasonable to think that " $n$ " will have negligible variations at temperatures lower than $T_{g}$, because in that region there is a very slight decrease in the density when the temperature increases. Assuming, therefore, that there is not appreciable variation in $\varepsilon$ and $n$ with temperature, except at $T_{g}$, in rigid or highly viscous systems, no blue spectral shift can be expected.

Taking into account the above mentioned, it seems that there is only one cause that could explain the red shifts observed in Fig. 1, which is the increase in the population of the upper vibrational levels of the DNS's electronic ground state. This effect would decrease the energy of the transition that gives the fuorescent emission.

Studies of polymeric systems where f uorescent probes or labels are immersed have revealed a sharp change in the tendency of the fluore cent intensity at a certain temperature, which usually is attributed to large variation in the refractive index of the system $[33,38]$. This phenomenon can be understood by considering the equation

$\phi_{F}=\frac{k_{F}}{k_{F}+k_{\mathrm{nr}}}=\frac{k_{F}^{0} n^{2}}{k_{F}^{0} n^{2}+k_{\mathrm{nr}}} \propto I$ (fluore cent intensity),

where $k_{F}$ and $k_{\mathrm{nr}}$ are the fuorescent and nonradiative constant rates, respectively, and $k_{F}^{0}$ is a constant independent of the temperature. Fig. 2 represents the natural logarithm of the fuorescent intensity with the inverse of temperature when the DNS is chemically attached to the epoxy matrix (sample APTES-B, open squares). Typical behavior in polymers can be observed [38], with a sharp change in the logarithm of the $\mathrm{fl}$ orescent intensity that usually is attributed to a polymer relaxation temperature, for instance a $T_{g}$. For the

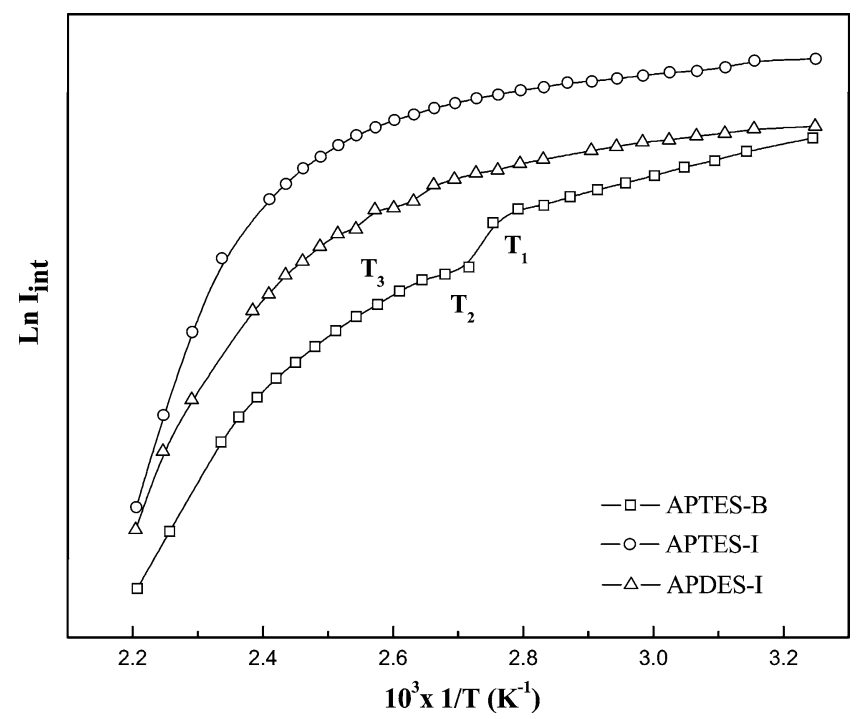

Fig. 2. Logarithm of f uorescent intensity as a function of temperature for the samples APTES-B, APTES-I, and APDES-I. sample APDES-B, a similar result can be assumed: the local DNS environment should be nearly the same, since equal epoxy conversion was reached in both samples (see experimental part).

In the low-temperature range (from 318 to $353 \mathrm{~K}$, below $T_{1}=353 \mathrm{~K}$ or $80^{\circ} \mathrm{C}$ ), there is a slight variation in the fl orescent intensity with temperature. In this temperature interval it can be assumed that $k_{F} \gg k_{\mathrm{nr}}$ and the fluore cent intensity dependence on temperature must be due mainly to refractive index changes (caused by electronic reorientation), which usually are very small. In the high temperature range (above $T_{3}=387 \mathrm{~K}$ or $115^{\circ} \mathrm{C}$ ), the fuorescent intensity has a more pronounced decrease when temperature increases because the contribution of the nonradiative processes to the DNS deexcitation becomes very important. Between $T_{1}$ and $T_{2}=368 \mathrm{~K}$ there is a sharp jump in the fluore cence intensity. If the matrix experiences a thermal transition with an important change in its refraction index, they should be translated into f uorescent intensity changes such as those shown in Fig. 2 for the interval $\left(T_{1}, T_{2}\right)$. Therefore, this results suggests that in this temperature interval a relaxation process of the system must exist, for example, a glass transition [38].

In Fig. 3 (open squares) the f rst moment of the emission band, $\langle v\rangle$, is represented as a function of temperature for the sample APTES-B. This representation shows more clearly the batochromic shift observed in the fuorescent emission spectra of Fig. 1. It is possible to see three zones: (i) between 30 and $85^{\circ} \mathrm{C}$ an approximately linear decrease in $\langle v\rangle$ is observed with temperature; (ii) between 85 and $100^{\circ} \mathrm{C}$ there is a stabilization in the $\langle v\rangle$ value; and (iii) above $100^{\circ} \mathrm{C}\langle v\rangle$ again decreases nearly linearly with temperature, although now with a higher slope than in the low temperatures range.

In the low range of temperatures, between 30 and $85^{\circ} \mathrm{C}$, the polymer is a glass and therefore, as explained before, the continuous decrease in the $\langle v\rangle$ value with temperature may be due to a higher population of the upper vibrational levels of the DNS's electronic ground state. Between

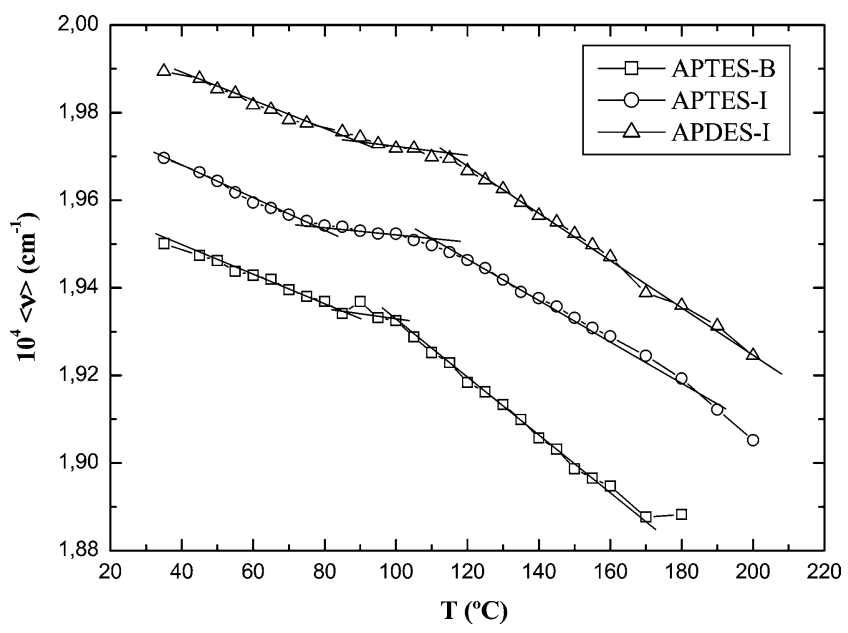

Fig. 3. First moment of the DNS emission band, $\langle v\rangle$, as a function of temperature for the samples APTES-B, APTES-I, and APDES-I. 
85 and $100^{\circ} \mathrm{C},\langle v\rangle$ is nearly constant. This result suggests that there must be an opposite effect, in terms of spectral shift, that compensates for the population increase of the upper vibrational levels (thermal activation). This effect may be due to that observed in low-viscosity solvents $[28,29]$ (hypsochromic shift, when the temperature increases due to changes in the properties of the solvent, $n$ and $\varepsilon$ ). In general, the glass transition phenomenon assumes a sharp variation in the polymer density and, thus, in the refraction index value. Therefore, in that range of temperatures, there can be considerable changes in the properties of the medium, which would imply blue shifts against the trend imposed by the thermal activation. This fact would explain the very small variation in the $\langle v\rangle$ value.

Finally, at temperatures higher than $100^{\circ} \mathrm{C},\langle v\rangle$ again decreases with temperature, although faster than in the frst interval considered. This result might represent a higher stabilization of the excited state. At temperatures above $T_{g}$, the free volume fraction in the polymer is higher, the density decreases, the dipole reorientation process is favored (shorter times), and therefore the probability of the DNS's excited state being stabilized by dipolar coupling increases greatly [33].

It is interesting to emphasize that the range of temperatures in which $\langle v\rangle$ is nearly constant perfectly matches the range in which the fluore cent intensity shows a sharp decrease, indicating that both photophysical parameters (integrated intensity and $\langle v\rangle$ ) are sensitive to the same change in the system, a thermal transition.

Taking into account that fuorescence gives local information, when the DNS is exclusively bonded to the epoxy matrix (Figs. 2 and 3, open squares), the temperature interval of change for the photophysical parameters studied should yield information equivalent to that obtained by any conventional technique such as DSC. Therefore, a conf rmation that the thermal transition observed by f uorimetry corresponds to a real glass transition could be to carry out a typical DSC experiment with the same sample. Fig. 4 represents the DSC

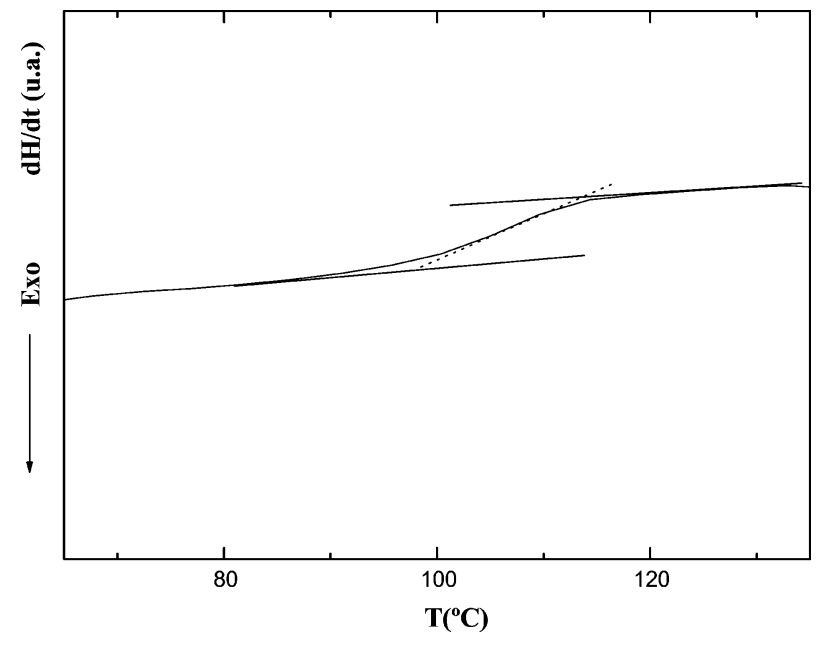

Fig. 4. DSC thermogram for the APTES-B sample. trace obtained for the APTES-B sample. It is observed that the heat capacity change assigned to the glass transition appears between 85 and $120^{\circ} \mathrm{C}(358-393 \mathrm{~K})$, the inf ection point being $T_{g}=107^{\circ} \mathrm{C}$. It is interesting, therefore, to emphasize the extraordinary concordance between the results obtained using the fuorescenct method and the DSC. This result seems to confir that the large changes with temperature observed in DNS fluore cence (intensity and $\langle v\rangle$ ) can be assigned to a real glass transition.

\subsection{Comparison between thermal transitions in the epoxy bulk and at the interface}

In Fig. 2 (open circles and triangles) is represented the natural logarithm of the f uorescent integrated intensity with the inverse of the absolute temperature when the DNS is chemically attached to the interface, samples APTES-I and APDES-I, respectively. It is observed that when the fuorescent label is located at the interface, the fuorescent integrated intensity decreases homogeneously, without jumps, when temperature increases. However, a more detailed observation seems to ref ect a very slight discontinuity whose temperature interval cannot be discerned. On the other hand, as described before, when the DNS is chemically bonded to the epoxy bulk (open squares), there is a sharp change in the fluore cent intensity with temperature. In both cases, the observed changes may be due to a glass transition. However, these results suggest that the fuorescent intensity measurement does not seem to be an eff cient parameter for obtaining thermal transitions at the interface, at least in this kind of systems.

In Fig. 3 is represented the $\mathrm{f}$ rst moment of the emission band as a function of temperature for the samples APTES-I (open circles) and APDES-I (open triangles). It can be observed that the curve prof les are independent of the localization of the DNS label. Furthermore, there is a temperature interval in every case where the frst moment almost does not change. These intervals, as we explained before, can be assigned to the respective glass transitions.

In Table 2 are summarized, for every sample studied, the limits of the temperature intervals in which the frst moment of the f uorescent band is constant with temperature. There are two facts that can be pointed out:

Table 2

limits of the temperature intervals in which the $\mathrm{f}$ rst moment of the f uorescence band is constant with temperature and $t_{g}$ 's obtained by dsc for the samples studied

\begin{tabular}{lll}
\hline Sample & $T_{\text {initial }}-T_{\text {fin } 1}\left({ }^{\circ} \mathrm{C}\right)$ & $\begin{array}{l}T_{g}\left({ }^{\circ} \mathrm{C}\right) \\
\text { (inflectio point) }\end{array}$ \\
\hline APTES-B & $85-100$ & 107 \\
APDES-B & - & 106 \\
APTES-I & $80-108$ & 97 \\
APDES-I & $90-115$ & 97 \\
\hline
\end{tabular}




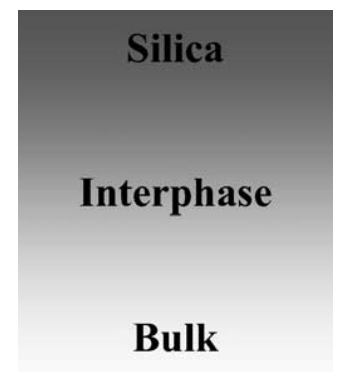

Scheme 1.

(i) On the average, the thermal transition occurs at higher temperatures when the DNS is located at the interface than when it is in the epoxy bulk.

(ii) The temperature interval in which the transition appears is larger when the f uorescence label is chemically bonded to the interface.

The frst observation suggests that the interfacial region is slightly more rigid than the epoxy bulk, which is entirely consistent with part of the work done by Lenhart et al. [16]. The second observation could be indicative of more pronounced heterogeneity. A possible explanation for the second observation could be found by considering the chain interpenetration model [2]. Throughout the coupling region the DNS must be located in different sites (with more or less rigidity and/or polarity) depending on the penetration of the matrix chains into the cross-linked network of the polysiloxane coating.

Scheme 1 illustrates the model of the interpenetrating polymer networks. The gray color gradient would ref ect the interpenetration chains gradient and therefore the heterogeneity in terms of different surroundings for the DNS label (different emission wavelengths). There is experimental evidence that throughout the interface there are structural and compositional, variations [39] that may cause the heterogeneity mentioned above.

On the other hand, in Fig. 3 it can be observed that the curve for the APTES-I sample is above the curve for the APTES-B sample. The average energy of the emission band is higher when the fuorescent label is attached to the coupling region independent of the temperature. There are two facts that could explain this result: (i) higher interfacial rigidity with less effective dipolar coupling (longer solvent relaxation time, blue shift) and (ii) less polarity in the interface with less stabilization of the excited state due to the dipolar interaction (blue shift). The fi st seems to be conf rmed by the values obtained from $\mathrm{f}$ uorescence for the glass transition temperatures (Table 2). The second would be in good agreement with the estimated solubility parameters for the epoxy resin and the polysiloxane. The solubility parameters are usually used to gain a comparative idea of polarity. Generally, a higher value of the solubility parameter is indicative of a higher polarity [32]. One way to estimate the solubility parameters is by means of the group contribution method [40]. In the case of DGEBA and the polysiloxane (polyAPTES) the following were obtained: $\delta_{\text {DGEBA }}=21 \mathrm{MPa}^{1 / 2}$ and $\delta_{\text {poly-APTES }}=17 \mathrm{MPa}^{1 / 2}$, respectively. From this estimation, the DNS label must be in a more polar environment when it is attached to the DGEBA. Therefore, in the DGEBA, the excited state stabilization should be more effective due to a higher dipolar interaction. This stabilization mechanism would also justify what can be observed in Fig. 3: the emission of DNS chemically bonded to the epoxy bulk is always red-shifted in relation to that coming from the DNS attached to the interface.

\subsection{Coating effect}

In this section, the results of f uorescence obtained when the DNS is chemically bonded to two differently modif ed silica particles (samples APTES-I and APDES-I) will be compared. The structural differences in the interface are due to the type of silane used to modify the surface of the silica particles.

In Fig. 3 the value of the photophysical parameter $\langle v\rangle$ as a function of temperature is represented for the samples APTES-I (open circles) and APDES-I (open triangles). In both cases, the same prof le is observed over the whole temperature range studied. The value of $\langle v\rangle$ when APTES is used to coat the silica particles is lower independent of the temperature. These differences seem to be exclusively due to differences in rigidity. If one considers that the two silanes used for coating should have very similar chemical characteristics, it is reasonable to assume that the contributions to the DNS fluore cent emission, that arise from the solvent properties should be very similar. Therefore, a higher energy for the f uorescent emission band or a blue shift in the sample APDES-I with respect to the sample APTES-I suggest more rigidity of the interface in the former.

From Fig. 3 it is also possible to extract the $T_{g}$ values for the samples APTES-I and APDES-I (Table 2). It can be observed that: (i) On average, the $T_{g}$ obtained with the APTES coating $\left(80-108^{\circ} \mathrm{C}\right)$ is lower than that obtained with the APDES coating $\left(90-115^{\circ} \mathrm{C}\right)$ and (ii) the thermal interval in which the fi st moment, $\langle v\rangle$, is kept constant is approximately the same for both samples. The f rst observation could be indicative of higher $f$ exibility for the interface generated with APTES, being in accordance with the value of $\langle v\rangle$ always being lower than that obtained when the interface comes from the APDES coating. The second observation suggests that in both cases the heterogeneity associated to the interface must be similar.

The APTES (trifunctional monomer) should generate a cross-linked polyorganosiloxane layer on the surface of the silica particles, while for the APDES (difunctional monomer) this is not expected. Generally, when the chemical structure is similar a higher cross-link density leads to a higher rigidity. However, the obtained results seem to indicate the opposite. A possible explanation to this apparent contradiction is to consider that with APTES, although 
with more cross-link density, a more open structure is obtained [41].

Other interpretations of the results may be based on greater epoxy chain interpenetration into the coupling region when the silica particles are coated with APDES. A lower cross-linking density in the polysiloxane coating layer should favor epoxy chain penetration, therefore facilitating the epoxy group reaction with the amino groups of the polysiloxane and consequently generating a highly crosslinked silane-epoxy network with more rigidity.

\section{Conclusions}

In this work, it has been demonstrated that the fuorescent response from labels can be used to study thermal relaxation of polymers at specif c sites. Due to this, differences have been found between apparent $T_{g}$ 's at the interface and in the epoxy matrix of a silica/epoxy system. Two photophysical parameters were selected to analyze the data: the integrated fluore cent intensity and the frst moment of the emission band. Although the fuorescent intensity was sensitive enough to determine an apparent glass transition in the epoxy bulk, the analysis when the fuorescence came from the interface was not so successful. However, the frst moment of the emission band was very useful in determining glass transitions independent of the local site (interface or epoxy bulk). Furthermore, the fuorescence results, interpreted in terms of the model of interpenetrating polymer networks, suggest that (i) independent of the silane coating, the interfacial region is slightly more rigid and heterogeneous than the epoxy bulk and (ii) the interface generated with APTES seems to be more fexible than that obtained with APDES.

\section{Acknowledgments}

The authors gratefully acknowledge projects of Epoxil (MAT2000-0391-P4-02) and Fibrodont (MAT2001-0677P3) for f nancial support.

\section{References}

[1] N. Suzuki, H. Ishida, Macromol. Symp. 108 (1996) 19.

[2] E.P. Plueddeman, Silane Coupling Agents, Plenum, New York, 1991.

[3] H. Hamada, N. Ikuta, N. Nishida, Z. Maekawa, Composites 25 (1994) 512.

[4] A.T. Dibenedetto, P.J. Lex, Polym. Eng. Sci. 29 (1989) 543.

[5] E.T. Vandenberg, L. Bertilsson, B. Liedberg, K. Uvdal, R. Erlandsson, H. Elwing, I. Lundström, J. Colloid Interface Sci. 147 (1991) 103.
[6] N. Ikuta, Z. Maekawa, H. Hamada, H. Ichihashi, E. Nishio, I. Abe, Controlled Interphases in Composite Materials, Elsevier, New York, 1990.

[7] G. Van Assche, B. Van Mele, Polymer 43 (2002) 4605.

[8] R.F. Brady, J.M. Charlesworth, Prog. Org. Coatings 24 (1994) 1.

[9] C.C. White, K.B. Migler, W.L. Wu, Polym. Eng. Sci. 41 (2001) 1497.

[10] J.L. Lenhart, J.H. van Zante, J.P. Dunkers, R.S. Parnas, Macromolecules 34 (2001) 2225.

[11] Y. Hou, A.M. Bardo, C. Martínez, D.A. Higgins, J. Phys. Chem. B 104 (2000) 212.

[12] T.D.Z. Atvars, E. Sabadini, S.M. Franchetti, Eur. Polym. J. 29 (1993) 1259.

[13] M. Talhavini, T.D.Z. Atvars, O. Schurr, R.G. Weiss, Polymer 39 (1998) 3221.

[14] M.R. Vigil, J. Bravo, T.D.Z. Atvars, J. Baselga, Macromolecules 30 (1997) 4871.

[15] C.J. Ellison, S.D. Kim, D.B. Hall, J.M. Torkelson, Eur. Phys. J. E 8 (2002) 155.

[16] J.L. Lenhart, J.H. van Zanten, J.P. Dunkers, R.S. Parnas, Macromolecules 34 (2001) 2225.

[17] J. González-Benito, F. Mikeš, J. Baselga, H. Lemetyinemm, J. Appl. Polym. Sci. 86 (2002) 2992.

[18] F. Mikeš, J. González-Benito, B. Serrano, J. Bravo, J. Baselga, Polymer 43 (2002) 4331.

[19] W. Rettig, Angew. Chem. Int. Ed. Eng. 25 (1986) 971.

[20] A.A. Ayuk, W. Rettig, E. Lippert, B. Bunsenges, Phys. Chem. 85 (1981) 553.

[21] F. Volmer, W. Rettig, E. Birckner, J. Fluorescence 4 (1994) 65.

[22] D. Olmos, A.J. Aznar, J. Baselga, I. Mondragón, J. González-Benito, submitted for publication.

[23] J. March, Advanced Organic Chemistry: Reactions, Mechanisms, and Structure, Wiley, New York, 1985.

[24] Haulang, in: K.D. Larison (Ed.), Molecular Probes: Handbook of Fluorescent Probes and Research Chemicals, 1992.

[25] J. González-Benito, F. Mikes, J. Bravo, A.J. Aznar, J. Baselga, J. Macromol. Sci. Phys. B 40 (3-4) (2001) 429.

[26] J. Mijović, S. Andjelić, Macromolecules 28 (1995) 2787.

[27] N. Poisson, G. Lachenal, H. Sautereau, Vib. Spectrosc. 12 (1996) 237.

[28] P. Suppan, J. Photochem. Photobiol. A Chem. 50 (1990) 293.

[29] P. Suppan, N. Ghoneim, Solvatochromism, The Royal Society of Chemistry, London, 1997.

[30] K. Fisher, D. Freiburg, Polym. Prepr. 29 (1988) 501.

[31] K. Ficht, K. Fisher, H. Hoff, C.D. Eisenbach, Makromol. Chem. Rapid Commun. 14 (1993) 515.

[32] J. González-Benito, A.J. Aznar, J. Baselga, J. Fluoresc. 11 (2002) 307.

[33] J.R. Lakowicz, Principles of Fluorescence Spectroscopy, Plenum, New York, 1999.

[34] R.S. Becker, Theory and Interpretation of Fluorescence and Phosphorescence, Wiley-Interscience, New York, 1980.

[35] J.F. Bristow, D.S. Kalika, Polymer 38 (1997) 287.

[36] B. Lestrirz, A. Maazauz, J.F. Gerard, H. Santereau, G. Boiteux, G. Seytre, Polymer 39 (1998) 6733.

[37] C. Beaucage, R. Composto, R.S. Stein, J. Polym. Sci. Part B Polym. Phys. 361 (1993) 319.

[38] A.P. Dorado, Movilidad de nudos en una red, Doctoral thesis, F. CC. Químicas U.N.E.D., 1995, p. 138.

[39] F.R. Jones, Key Engine Mater. 116-117 (1996) 41.

[40] J. Brandrup, E.H. Immergut, E.A. Grulke, in: Polymer Handbook, Wiley, New York, 1999, chap. VII, p. 675.

[41] J. González-Benito, A.J. Aznar, A. Maçanita, J. Baselga, Bol. Soc. Esp. Cer. Vidrio 39 (2000) 396. 